\title{
IMPLANTACIÓN DE LA AGENDA 21 LOCAL EN EUROPA: DIVERGENCIAS EN RITMOS Y ENFOQUES ${ }^{1 *}$
}

\author{
I. Aguado Moralejo \\ Universidad del País Vasco/Euskal Herriko Unibertsitatea \\ Facultad Filología y Geografía e Historia \\ Francisco Tomás y Valiente, s/n. Edificio Anejo, Dpcho. 23, $1^{\text {a }}$ planta \\ Apartado 2111 - 01006 Vitoria-Gasteiz - Tel.: 945013989 \\ itziar.aguado@ehu.es \\ C. Echebarria Miguel \\ Universidad del Pais Vasco/Euskal Herriko Unibertsitatea \\ Facultad de Ciencias Económicas y Empresariales \\ Avda. Lehendakari Aguirre, 83 - 48015 Bilbao \\ Tels.: 946013887 - 946018096 - Fax: 946017087 \\ carmen.etxebarria@ehu.es \\ J. M. Barrutia Legarreta \\ Universidad del País Vasco/Euskal Herriko Unibertsitatea \\ Facultad de Ciencias Económicas y Empresariales \\ Avda. Lehendakari Aguirre, 83 - 48015 Bilbao \\ Tels.: 946013887 - 946018096 - Fax: 946017087 \\ josemaria.barrutia@ehu.es
}

\begin{abstract}
Resumen: El artículo tiene por objeto presentar un análisis de la implantación de la A21L en Europa. Nos centraremos, especialmente, en los países de Europa Occidental, al considerarlos los más avanzados en la implementación de este tipo de políticas. La investigación permite constatar, por un lado, el gran auge que se está dando a la difusión de los procesos de A21L y, por otro, las grandes diferencias regionales en el ritmo de desarrollo y enfoque de los programas de A21L.
\end{abstract}

Palabras clave: Agenda 21 Local, Desarrollo Sostenible, situación europea.

\footnotetext{
* Recibido: 22-06-06. Aceptado: 11-05-07.

I Este trabajo de investigación ha sido realizado gracias al apoyo de la UPV (Subvención General a Grupos de Investigación): 9/UPV 00032.321-15908/2004 y de la Cátectra UNESCO sobre "Desarrollo Sostenible y Educación Ambiental": Código OTRI: UNESCO 03/05.
} 
Summary: The article takes as an object to present an analysis of the implantation of the A21L in Europe. We centre, especially, in the countries of Western Europe yet they are the leaders of these processes. The investigation allows to state, on one hand, the high diffusion of the processes of $\mathrm{A} 21 \mathrm{~L}$ across the planet and on the other hand, the wide regional differences in the rhythm of development and approach of the programs of $\mathrm{A} 21 \mathrm{~L}$.

Keywords: Local Agenda 21, Sustainable Development, European Situation.

\section{Introducción}

Es un hecho notorio, el creciente reconocimiento que se le está dando a la A21L, ya que en los últimos años está siendo fuertemente impulsada por los diversos Gobiernos Nacionales, al tiempo que adoptada por numerosos municipios a lo largo y ancho del planeta. Ahora bien, con frecuencia, la A21L ha sido implementada bajo distintos nombres, principalmente, como Planes de Mejora Ambiental o como Planes de Sostenibilidad, pero la idea que subyace bajo estas diferentes denominaciones se asienta en la metodología y en los objetivos propuestos por la Conferencia de las Naciones Unidas sobre Medio Ambiente y Desarrollo, de 1992. Otras muchas veces, su implantación se realiza a través de programas de asistencia establecidos a nivel internacional, como el Programa de Ciudades Sostenibles de las Naciones Unidas, el Programa Capacity 21 o el Programa de Gestión Ambiental Urbana del ICLEI (International Council for Local Environment Initiatives, 1997, 2002). Por todo ello, intentar comparar las distintas iniciativas desarrolladas a nivel internacional resulta muy complicado debido a que, como señalan Lafferty y Eckerberg (1998), existe una gran confusión acerca de las implicaciones reales de la A21L, sobre todo si atendemos a los Informes Nacionales que se han venido realizando con objeto de la celebración de las distintas Cumbres sobre Desarrollo Sostenible de las Naciones Unidas. De ellos se desprende que muchos gobiernos identifican toda acción que mejore la calidad ambiental o que implique la consecución de un Desarrollo Sostenible con la A21L.

En consecuencia, en el presente artículo vamos a presentar la situación de la A21L en Europa. Nos centraremos, sobre todo, en los países de Europa Occidental, que pueden ser calificados como líderes a nivel mundial en relación con la A21, puesto que han desarrollado numerosas iniciativas relacionadas con la protección ambiental, aunque con grandes diferencias en el ritmo de desarrollo y enfoque. 


\section{La Agenda 21 Local: Marco Conceptual}

La Agenda 21 Local es el resultado de las diversas iniciativas desarrolladas por las Autoridades Locales en apoyo a la Agenda 21, un Plan de Acción Global para el Desarrollo Sostenible que fue aprobado por 173 gobiernos en la Conferencia de las Naciones Unidas sobre Medio Ambiente y Desarrollo, celebrada en Río de Janeiro en 1992, con el objetivo de conciliar los temas relacionados con el medio ambiente y el desarrollo, reflejando un consenso y un compromiso político al más alto nivel para la consecución de un desarrollo sostenible (Lafferty, 2001; Hewitt, 1998). Para ello, la A21L propugna un desarrollo sostenible descentralizado pero concertado, en el que cada ciudad ha de tener autonomía para autoorganizarse y para establecer proyectos de actuación y modelos de gestión propios. Esto es, esas políticas han de estar instauradas en un marco espacial más amplio y han de ser coherentes con el resto de las políticas de los niveles administrativos superiores, convirtiéndose, de este modo, en el instrumento que aspira a contener las actuaciones locales que intentan trasladar la concepción global de sostenibilidad, desde la retórica política a nivel global a la esfera operativa y real a la escala local (Font y Subirats, 2000).

El movimiento de las AL21 está siendo especialmente impulsado por el ICLEI (Internacional Council for Local Environmental Initiatives), institución fundada en 1990 por más de 200 autoridades locales, la mayoría de las cuales son europeas o estadounidenses. Funciona como una red de autoridades locales que permite el intercambio de experiencias entre ciudades difundiendo ejemplos de buenas prácticas ambientales a nivel mundial. Entre las acciones que ha acometido este organismo, destaca la elaboración de la Guía Europea para la planificación de las Agendas 21 Locales (Hewitt, 1998), documento que sirve de base metodológica para la implantación de la AL21, puesto que aunque la implantación de la Agenda 21 Local debe ser un proceso consensuado, que parta de las particularidades y características propias de cada municipio, podemos concluir que, en el plano operativo, la metodología propuesta por el ICLEI es la más empleada habitualmente.

\section{Perspectiva Mundial de la Agenda 21 Local}

Ya hemos señalado la dificultad de comparar las distintas actuaciones en temas de A21L que se están implementando en el planeta. Aún así, un elemento de referencia muy valioso son los distintos Informes de Evaluación publicados por el ICLEI. El primero de ellos fue presentado en la reunión de la "Cumbre Río+5" celebrada en 1997 y, el segundo, en la Cumbre Mundial sobre Desarrollo Sustentable de la ONU celebrada en Johannesburgo en 2002. Para la redacción de este último, se remitió un. 
cuestionario a más de 1.800 gobiernos locales con el fin de identificar las áreas que se estaban priorizando en los distintos procesos de A21L desarrollados a nivel internacional. En total, participaron 633 gobiernos locales individuales y 146 asociaciones de gobiernos locales, representando a 113 países. Las principales conclusiones se concretaron en los siguientes puntos:

- 6.416 autoridades locales en 113 países se encontraban en el año 2002 en proceso de elaboración de un proyecto de A21L o se habían comprometido a ello.

- Hasta entonces, se habían realizado Campañas Nacionales en 18 países, contabilizando 2.640 procesos de A21L.

- En el 73\% de los municipios con A21L se habían establecido grupos de agentes formales.

- La A21L aparecía integrada, dentro del sistema municipal, en el 59\% de los municipios.

- La gestión del agua destacaba como una prioridad común para los municipios de todas las regiones del mundo, con independencia de su situación económica.

- Todos los municipios, demandaban un mayor impulso de las administraciones centrales y denunciaban el insuficiente apoyo financiero.

Gracias a esta evaluación se pudo percibir el gran auge que se estaba dando a la difusión de los procesos de A21L, a través del planeta. Sin embargo, también se confirmaron las grandes diferencias regionales en el desarrollo de los programas de A21L en el mundo, destacando Europa como la región que más municipios tenía en procesos de A21L, frente al amplio desinterés mostrado en África. De este modo, mientras que en África el objetivo principal de las Agendas 21 Locales se situaba en la lucha contra la pobreza, en Europa se evidenció la patente preocupación por los problemas ambientales. Asimismo, se puso de manifiesto la importancia del desarrollo de Campañas Nacionales, frecuentes en Europa (ocho países con campañas nacionales en el año 2002, que aglutinaron un total de 2.011 procesos de A21L) y en la región de Asia-Pacífico (seis países con campañas nacionales activas, que representaban a más del 75\% de los procesos de A21L en desarrollo), y prácticamente inexistentes en otras regiones, en el proceso de difusión y adopción de la Agenda 21 (Véase Tabla 1).

Tabla 1. Desarrollo de Campañas de Agenda 21 a nivel nacional.

\begin{tabular}{ll}
\hline Región & Países con Campañas Nacionales \\
\hline Europa & Alemania, Finlandia, Islandia, Irlanda, Italia, Noruega, Suecia y Reino Unido \\
Norte América & Ninguno \\
América Latina & Ecuador y Perú \\
África & Sudáfrica \\
Oriente Medio & Turquía \\
Asia y Oceanía & Australia, China, Japón, República de Corea, Mongolia y Sri Lanka \\
\hline
\end{tabular}

Fuente: Elaboración propia. 


\section{Europa: Líder a Nivel Mundial}

\subsection{Una visión de conjunto}

Todos los estados miembros de la Unión Europea (UE-15) se comprometieron en la Cumbre de Río de Janeiro de 1992, a desarrollar la Agenda 21. En efecto, en base a ese compromiso la mayor parte de los países europeos ha establecido medidas de apoyo desde los gobiernos nacionales tales como el Desarrollo de Campañas Nacionales de A21L, la Elaboración de guías metodológicas, la Creación de redes de autoridades locales, la Organización de Cursos de Formación, la Construcción de páginas web que tratan sobre Desarrollo Sostenible, etc. De este modo, y como evidencia la última evaluación realizada por ICLEI en el año 2002 a nivel mundial, la A21L ha sido tomada muy en serio en Europa, puesto que más de 5.000 gobiernos locales europeos habían iniciado o desarrollado para entonces procesos de A21L. En ese notable desarrollo y compromiso con la A21L, ha jugado un papel crucial la Campaña Europea de Ciudades y Pueblos Sostenibles, celebrada por primera vez en Aalborg (Dinamarca), en 1994 y que contaba con más de 2.200 autoridades locales a finales del año 2005.

De acuerdo con Lafferty (2001), se pueden diferenciar tres grupos de países en Europa Occidental. Por un lado, se situarían Suecia, Reino Unido y Holanda, países que respondieron rápidamente a la llamada de la Cumbre de Río y cuyos Gobiernos Nacionales impulsaron desde el principio, y de manera muy significativa, los Programas de Agenda 21. A estos países, les siguieron el resto de los países escandinavos (Dinamarca, Noruega y Finlandia), que aunque empezaron más tarde si sí manifestaron un elevado compromiso por parte de las autoridades locales. Por otro lado, se situarían Alemania y Austria, cuya respuesta fue aún más tardía y donde el seguimiento de la A21L por parte de las autoridades locales ha sido más bien escaso. En último lugar, se situarian los países mediterráneos (Italia, Portugal, España y Francia), que junto con Irlanda empezaron a mostrar interés por la Agenda 21 Local a finales de los años noventa.

\subsection{Análisis por países de la Agenda 21 Local}

\subsubsection{Suecia}

Suecia se puede considerar como el país pionero en implantar la A21L, puesto que la rápida difusión de la Agenda 21, fundamentalmente, a escala local, donde los 288 
municipios suecos se han comprometido a adoptarla, le ha convertido en un referente a nivel europeo y mundial. En este sentido, el Gobierno Central ha jugado un papel primordial, muy especialmente, a través de la transmisión de información a los municipios y mediante la elaboración, en el año 1995, de la Estrategia de Desarrollo Sostenible (Löffler y Payne, 1999). Además, en ese mismo año, constituyó el Comité Nacional de la Agenda 21 con el objeto de elaborar, anualmente, un informe de situación de la implantación de la A21L, mientras que en Junio de 1999, estableció el Centro Sueco para la Sostenibilidad Ecológica, el Swecol, una entidad destinada a la coordinación de la A21L a nivel nacional.

Otra razón adicional, que justifica la pronta actividad por parte de los municipios suecos, descansa en el alto grado de autonomía del que gozan las autoridades locales (Adolfsson, 2002). Asimismo, en muchas ocasiones, los Ayuntamientos han sido los encargados de implementar las políticas instauradas desde el Gobierno Central, relacionadas con la protección medioambiental. Es necesario también resaltar el activo papel desempeñado por las ONGs en relación a la difusión de la Agenda 21, teniendo un buen ejemplo de ello en la Asociación para la Agenda 21 y el Desarrollo Sostenible en Suecia, organización en la que participan los municipios, el sector empresarial, diferentes ONGs y el Ministerio de Medio Ambiente del Gobierno Sueco.

Otro aspecto no menos importante, por ser una de las principales barreras para la implementación de la A21L, en el que se ha avanzado, notablemente, es el de la financiación. En este sentido, desde 1998, se articulan toda una serie de ayudas dentro del Programa de Inversión Local (Lokala Investerings Programmet, LIP), para el apoyo de la A21L en los municipios a través de la formación de la ciudadanía y de la articulación de la participación ciudadana en los procesos de A21L (Adolfsson, 2002). En total, para el periodo 1998-2003, se destinaron 6,2 billones de coronas suecas, que cubrían el $30 \%$ de las inversiones realizadas por las autoridades locales, que hacian frente al 70\% restante de la financiación (Eckerberg et al., 2003).

En definitiva, podemos afirmar que la fuerte autonomía local junto con el apoyo financiero, técnico y político del Gobierno Central sueco son los principales factores que han llevado a Suecia a ser considerada una nación líder en la implantación de la A21L. Junto a ello, el hecho de que las autoridades locales cuenten también con una notable cantidad de recursos de todo tipo, ha beneficiado al proceso de difusión de la A21L (Khakee, 2002). Un factor adicional que ha influido favorablemente es la amplia cultura ambiental de los ciudadanos, así como la larga trayectoria del país en el desarrollo de políticas ambientales (Gomila, 2000). Por último, de los datos del análisis de Eckerberg et al. (2003), se deduce que en los últimos años está retrocediendo el interés tanto por parte de los políticos como de la ciudadanía en relación a la A21L. Ahora bien, estas mismas autoras afirman también que se están clesarrollando otro tipo de acciones, que aunque no se denominen A21L, sí implementan, igualmente, el Desarrollo Sostenible. 


\subsubsection{Reino Unido}

El Reino Unido sorprendió al resto de los países por su pronta respuesta a la $\mathrm{A} 21 \mathrm{~L}$, ya que la primera Estrategia Nacional de Desarrollo Sostenible fue presentada en el año 1993, mientras que la segunda Estrategia fue aprobada en el año 1999. En esta última, se puso de relieve el creciente compromiso por parte del gobierno laborista respecto a la $\mathrm{A} 21 \mathrm{~L}$, que ha dado lugar a que sea adoptada por numerosos municipios y condados. En efecto, el Primer Ministro Tony Blair, alcanzó en 1998 un Acuerdo con los gobiernos locales para ayudarles a desarrollar sus estrategias de A21L. En contrapartida, las autoridades locales se comprometieron a desarrollar su propia A21L, para el año 2001, destinando tiempo, personal y recursos para este fin. Adicionalmente, para el año 2001, las regiones británicas se comprometieron a abordar sus Estrategias de Desarrollo Sostenible.

Pero, en contra de lo que cabría esperar, en ningún caso se articularon ayudas financieras con estos propósitos ni se detalló ninguna ley que obligase a las autoridades locales a implantar la A21L. Por el contrario, lo que sí se hizo fue proporcionar material documental y cursos de formación a los líderes políticos y administrativos (Löffler y Payne, 1999). En base a ello, se puede afirmar que el Reino Unido, al igual que la mayoría de los gobiernos europeos, ha demostrado un notable compromiso nominal en relación a la $\mathrm{A} 21 \mathrm{~L}$, que no siempre se ha plasmado en un compromiso práctico (Jonas et al., 2004). A pesar de ello, conviene señalar que hacia mediados de los años noventa, la mayor parte de las autoridades locales estaban inmersas en procesos de A21L, si bien, como indican Jonas et al., (2004), estos procesos no destacaban por la calidad de sus estrategias.

Con el objetivo de contribuir a mejorar la calidad de las estrategias de A21L, la Federación de Gobiernos Locales (Local Government Management Board, LGMB), realizó numerosos estudios relacionados con el problema de la participación ciudadana, la metodología de la A21L, el desarrollo de indicadores de sostenibilidad, etc. Este organismo, derivó, en el año 1999, en la Agencia de Mejora y Desarrollo (Improvement and Development Agency, I\&Dea), erigiéndose ésta como la principal institución pública en asuntos relacionados con la A21L.

Estrechamente relacionado con la problemática de la evaluación, se encuentra el desarrollo de Indicadores de Sostenibilidad, sobre los cuales se ha trabajado de manera muy intensa en el Reino Unido. Junto a ello, se han desarrollado indicadores específicos de A21L por parte del gobierno central, en colaboración con los gobiernos locales, que han sido adoptados por treinta Ayuntamientos. También, numerosos Ayuntamientos como Kirklees, Mendip, Lancanshire o Leicester, entre otros, están desarrollando metodologías participativas, siendo éste, quizás, éste uno de los aspectos en los que mejor son valorados los procesos de A21L por parte de otros países (Church y Young, 2001; Wild y Marshall, 1999). 
Para finalizar, podemos afirmar que la A21L se ha contemplado en el Reino Unido como un instrumento para la modernización ecológica, dado que, tradicionalmente, han sido los departamentos de medio ambiente de los Ayuntamientos quienes han asumido su preparación y puesta en marcha (Bond et al., 1998). No obstante, y en contradicción con las medidas introducidas para la modernización de la gestión local, en los últimos años, parece ser que la A21L está siendo relegada del plano político por numerosas autoridades locales. Como afirman Jonas et al. (2004), esto, puede ser causado en parte por la adopción de otros programas sustitutivos, pero, sobre todo, por la falta de un entramado legislativo sólido.

\subsubsection{Holanda}

Holanda, cuenta con una gran tradición en la elaboración de Políticas Ambientales y de concienciación en torno al Desarrollo Sostenible. De hecho, fue el primer país del mundo en introducir el concepto de Desarrollo Sostenible como objetivo específico en el Plan Ambiental Nacional de 1988. Junto a ello, el alto grado de desarrollo de la política ambiental municipal debería haber facilitado la implantación temprana, tras la Cumbre de Río, de la A21L en Holanda. Ahora bien, en contra de las previsiones, la consolidación de las Agendas 21 Locales en Holanda no se produjo hasta el año 1994. Según Gomila (2000), la explicación a esta pasividad inicial, se puede hallar en la percepción, tanto por el Gobierno Nacional como por los gobiernos locales, de que la A21L no aportaba un valor añadido a las políticas ambientales que ya se venían desarrollando. Sin embargo, en 1994, la situación cambia: se crea la Comisión Nacional de A21L, se establece apoyo financiero para que los municipios instauren la A21L y el Segundo Plan de Política Ambiental Nacional promociona la A21L, así como su utilidad en la gestión local. A partir de entonces, el liderazgo en la implementación de la A21L en Holanda fue asumido, conjuntamente, por el Ministerio de Medio Ambiente (VROM), la Asociación Holandesa de Municipios (VNG) y el Comité Nacional de Cooperación Internacional y Desarrollo Sostenible (NCDO), formado por unas cincuenta ONGs (Coenen, 2001).

En. 1998, con la puesta en marcha del Tercer Plan de Política Ambiental Nacional, vuelve a darse un punto de inflexión en la implementación de la A21L en Holanda, al verse reducida la financiación a los Ayuntamientos. Por ello, no es extraño, que la trayectoria y las características de la A21L estén estrechamente vinculadas con la evolución y disponibilidad de los fondos. De acuerdo con Coenen (2001), la A21L en Holanda se caracteriza porque: 1) El contenido suele centrarse en aspectos relativos al medio ambiente o en proyectos en áreas como construcción sostenible o ahorro energético, 2) En general, las Agendas 21 Locales adoptan la forma de agenda de actividades a realizar en el marco municipal y no constituyen procesos visionarios, salvo en casos excepcionales, y 3) Presenta procesos de participación muy diversos, que comprenden desde consultas informales a plataformas estructurales de participación. 
De todas formas, cabe destacar la existencia de lo que podríamos denominar una amplia cultura de diálogo y de consenso, donde la participación no sólo se articula a través de las Instituciones formales sino también mediante canales informales.

Para concluir, falta añadir que aunque en el caso de Holanda, no se pueda hablar de una Estrategia de Desarrollo Sostenible propiamente dicha, existen, como hemos visto, un conjunto de programas y planes a nivel nacional que pudieran ser considerados como tales. El documento más reciente de este tipo aprobado en Julio de 2003 , Duurzame Daadkracht (Motivaciones para la Acción Sostenible), podría considerarse una Estrategia de Desarrollo Sostenible, pues intenta trasladar a las políticas nacionales el objetivo de alcanzar un Desarrollo Sostenible a largo plazo. Queda por ver, cuáles serán las repercusiones de dicho documento, ya que todavía no ha transcurrido el tiempo suficiente para observar si está consiguiendo modificar la trayectoria seguida hasta el momento.

\subsubsection{Noruega}

Noruega es otro de los países que ha dado un fuerte impulso a la Agenda 21, especialmente, en los últimos años, de tal manera que más de la mitad de los municipios han realizado ya sus Planes de Acción. En todo caso, para entender el amplio seguimiento que tiene la A21L en Noruega, es necesario mencionar la significativa Reforma Ambiental introducida por el Gobierno Central entre los años 1991 y 1997, mediante la cual se establecieron fondos públicos para permitir que los municipios pudieran designar un técnico ambiental, con los objetivos, por un lado de incrementar la capacitación en medio ambiente del personal de los Ayuntamientos, y, por otro, de intentar conseguir la integración del componente ambiental en el resto de políticas municipales (Aall, 2001). Esta medida, en un primer momento, ralentizó la implantación de la A21L, puesto que los esfuerzos eran dirigidos a la renovación institucional, pero, sirvió para que se consolidasen las bases necesarias sobre las que posteriormente se iba a erigir la A21L.

De este modo, la fecha clave en el desarrollo de la A21L en Noruega fue 1997, puesto que en dicha fecha finalizó la Reforma Ambiental y se constituyó un órgano de coordinación de la A21L a nivel nacional promovido por el Ministerio de Medio Ambiente y Energía. En poco tiempo, el auge de la A21L en los municipios noruegos se hizo evidente. En este mismo año, el Libro Blanco Environmental Policy for Sustainable Development, estableció como meta principal la implantación de la A21L. Igualmente, otro hito relevante fue la Conferencia de Fredrikstad celebrada en 1998, donde se aprobó la Declaración de Fredrikstad, que constituye la expresión noruega del contenido de la A21L, considerándose el punto de partida de los procesos de A21L por parte de los municipios. Todos los condados noruegos y el $60 \%$ de los municipios han ratificado dicha Declaración, comprometiéndose a movilizar a los ciu- 
dadanos, empresas y organizaciones en los procesos de A21L, a la integración del desarrollo sostenible en todos los sectores, a trabajar sistemáticamente por el desarrollo sostenible, con una visión de largo plazo, a integrar el desarrollo sostenible en la planificación, al empleo de indicadores y a promover el ahorro energético (Aall, 2001).

El papel del Gobierno Nacional ha sido notable desde 1998, aunque en un principio, se mostró reticente a financiar directamente proyectos de A21L. A pesar de ello, entre los años 1997 y 2001, destinó entre uno y dos millones de coronas noruegas anuales para la financiación de actividades locales, recursos administrados, principalmente, por los coordinadores de las Agendas 21 regionales. En esta línea, la Asociación Noruega de Autoridades Locales y Regionales ha impulsado también, intensamente, la A21L, tanto mediante el apoyo al establecimiento del ICLEI en 1990, como a través de la organización del trigésimo Congreso Mundial de la Unión de Autoridades Locales en 1991, en el cual se adoptó la Declaración de Oslo sobre Medio Ambiente, Salud y Calidad de Vida, que contribuyó, significativamente, al desarrollo del capítulo 28 de la Agenda 21.

En el año 2001, el gobierno realizo realizó otro encuentro, similar al de Fredrikstad, la Conferencia "Sinergy 21", con el objetivo de relanzar la A21L a nivel nacional. A pesar de ello, el gobierno suprimió la financiación a los municipios, la Secretaría de A21L se cerró y el Programa para el Estímulo de la A21L, instaurado tras la Conferencia de Fredrikstad, finalizó. Únicamente el Ministerio de Asuntos Exteriores se mostró activo en los años previos a la Conferencia de Johannesburgo del año 2002 y, en todo caso, éste tiene la misión de elaborar el Informe sobre el Grado de Avance de la A21L en Noruega.

En este contexto, una encuesta nacional realizada en el verano del año 2000 , mostró que cerca del 70\% de los municipios noruegos se habían comprometido a desarrollar su A21L. Sin embargo, en esa misma encuesta se evidenció cómo, en la práctica, eran pocos los Ayuntamientos que habían implantado el Plan de Acción o los que habían definido e integrado indicadores de sostenibilidad, por lo que se puso de manifiesto la brecha existente entre las ambiciones municipales y la realidad. De esta forma, se observa como, al igual que en Suecia, los compromisos de adopción de la A21L, no siempre se materializan (Aall, 2001).

Por todo ello, y con el objetivo de promover la A21L, en octubre de 2003, se lanzó la última Estrategia Nacional de Desarrollo Sostenible o Agenda 21 Nacional. Para el seguimiento de dicha Estrategia, se estableció un "Comité Verde" compuesto por varias secretarías estatales de los ministerios más directamente relacionados con la A21L, coordinados por el Ministerio de Finanzas y liderado por la Oficina del Primer Ministro. En cualquier caso, habrá que esperar un tiempo para comprobar si esta 
nueva estrategia sirve para relanzar los procesos de A21L en Noruega y devuelve este programa a la actualidad política del país.

\subsubsection{Dinamarca}

En el contexto danés, se puede encontrar un amplio abanico de experiencias y proyectos (pueblos ecológicos, actividades de educación ambiental, etc.), desarrollados desde la década de los setenta, que se podrían considerar dentro del campo de actuación de la A21L (Gram-Hanssen, 2000). Sin embargo, no será hasta la década de los años noventa, cuando surjan numerosos proyectos, propiamente de A21L, como resultado, fundamentalmente, del notable impulso facilitado por el Gobierno Central. Más en concreto, se puede considerar que en Dinamarca, se empezó a trabajar en A21L en el año 1994, cuando el Ministerio de Medio Ambiente y Planificación Territorial, la Asociación Nacional de Autoridades Locales y la Asociación Danesa de Condados y Concejos asumieron, conjuntamente, el liderazgo de la Campaña Nacional de la Agenda 21, que desembocó en la creación de una Red de A21L. A ello, le siguieron otras actuaciones como la publicación de una guía metodológica, la celebración del Día Nacional de la Agenda 21, el desarrollo de la planificación estratégica ambiental, etc.

Se debe señalar que es el Ministerio de Medio Ambiente, el que asume las competencias respecto a la Agenda 21, competencias materializadas en diversas actividades para promocionarla por todo el país (publicación de folletos y revistas acerca de la A21L, proyectos demostrativos, organización de jornadas, etc.). No obstante, la colaboración con el Ministerio de Asuntos Urbanos y Vivienda, a través de la realización desde 1999 del Plan de Acción The City of the Future, o con la Asociación Nacional de Autoridades Locales, es muy estrecha. En relación con esta última, la elaboración en el año 2001 de una encuesta para medir el nivel de avance que se estaba alcanzando en relación a la $\mathrm{A} 21 \mathrm{~L}$, mostró unos resultados muy positivos ya que se comprobó que el 75\% de los municipios estaban desarrollando la A21L y que las ayudas destinadas a iniciativas ciudadanas relacionadas con la A21L, por parte de los municipios, habían ascendido en un $40 \%$.

En cuanto a la financiación proveniente del Gobierno Nacional, cabe destacar los llamados Fondos Verdes, partida presupuestaria dependiente del Ministerio de Medio Ambiente que se destina al apoyo tanto directo como indirecto de proyectos de A21L. Anualmente, desde 1997, dentro de esta partida, se han venido destinando cincuenta millones de coronas danesas. Igualmente, se han destinado diez millones de coronas danesas anuales para financiar proyectos individuales de A21L. Por otra parte, con motivo de la celebración de la Cumbre de Johannesburgo, se presentó la Estrategia Nacional de Desarrollo Sostenible Shared Future Balanced Development en el año 2002. 
En resumen, Dinamarca ha ejercido, aunque con dos años de diferencia respecto a Suecia, un papel muy activo tanto a nivel municipal como por parte del Gobierno Nacional en la promoción e implantación de la A21L. En este rápido avance y difusión, ha influido el alto grado de autonomía municipal, la amplia trayectoria en política ambiental y los sólidos mecanismos de participación que caracterizan a la planificación local en Dinamarca. Sin embargo, parece ser que el éxito de la A21L no siempre es tan evidente, ya que, muchas veces, la A21L se utiliza como una etiqueta que se coloca a acciones que ya se venían realizando en la gestión local (Holm y Wambui Kamara, 2001).

\subsubsection{Finlandia}

En los primeros años de andadura tras la Cumbre de Río, el gobierno finlandés mostró una actitud menos entusiasta en relación a la A21L que la del resto de los países nórdicos, siendo pocos los municipios que se sumaron a la A21L. La respuesta del Gobierno Central a la Cumbre de Río se concretó, por un lado, en la publicación del documento Elaboración de las Opciones de Futuro de Finlandia: la implantación de la Agenda 21 en Finlandia y, por otro, en el establecimiento de la Comisión Nacional de Desarrollo Sostenible, todo ello, en el año 1993. Posteriormente, en el año 1995 , se aprobó el Programa de Acción Nacional denominado Finnish Action for Sustainable Development, mientras que en el año 1998, se constituyó la Comisión Nacional de Agenda 21, dirigida por el Primer Ministro, cuya misión es la de coordinar y promover la Agenda 21 en el nivel local, a través de la realización de Campañas Nacionales de A21L.

Sin embargo, uno de los aspectos más negativos de la actuación del Gobierno Central finlandés es la no concesión de ningún tipo de financiación a los municipios para la implantación de la A21L, asumiendo que son las autoridades locales quienes han de tomar la iniciativa. No obstante, se observa que la mayor parte de los Ayuntamientos no destina recursos económicos específicos para la A21L, sino que ha procedido a redimensionar las actividades ambientales municipales, de manera que han introducido nuevas formas de cooperación entre los sectores administrativos, las autoridades locales, las organizaciones y los ciudadanos (Frank et al., 2000). Otros agentes destacables que han tomado parte en la difusión de la A21L, han sido la Asociación de Regiones y Autoridades Locales Finlandesas y diversas ONGs, algunas de las cuales contribuyeron, ampliamente, al desarrollo de la Campaña Finland 21, en 1997.

En todo caso, se constata un cierto retraso por parte de Finlandia, respecto a sus homólogos nórdicos. Ello se puede achacar, en primer lugar, a la crisis económica de principios de los noventa. En segundo lugar, a la mayor centralización existente en Finlandia, en relación al resto de los países nórdicos, lo que determina que las auto- 
ridades locales dispongan de menos recursos para el impulso de la A21L. Y, en tercer lugar, podemos citar el todavía escaso grado de conocimiento que se tiene sobre la A21L en Finlandia, donde, a menudo, es identificada como algo abstracto que no implica una cooperación intersectorial en la gestión local ni una planificación participativa (Frank et al., 2000).

\subsubsection{Alemania}

Alemania ha mostrado una actitud proactiva en torno a la incorporación del Desarrollo Sostenible en sus Planes y Políticas Nacionales. Prueba de ello, fue la constitución, pocos meses después de la Cumbre de Río del año 1992, del Consejo Científico del Gobierno Federal sobre Cambios Medioambientales Globales encargado de proponer recomendaciones para que las políticas integrasen el objetivo del Desarrollo Sostenible. El siguiente paso, fue el inicio, en 1996, de un proceso de diálogo denominado "Pasos hacia el Desarrollo Sostenible" por parte del Ministro de Medioambiente. Las conclusiones de dicho debate, fueron introducidos en el documento titulado Borrador para un programa de Política Ambiental, que designó a la Agencia Federal del Medioambiente (Umweltbundesamt, UBA) responsable para la elaboración de la Estrategia Nacional de Desarrollo Sostenible, estrategia que fue finalmente aprobada en abril de 2002 (Heidbrink y Paulus, 2000).

Por el contrario, en el marco de la A21L, el Gobierno Federal, amparándose en el principio de la autonomía local como característica de base en el desarrollo de los programas de A21L, no empezó a actuar hasta el año 1998. En dicho año, lideró un estudio sobre la efectividad de los planes de las Agendas 21 municipales para el desarrollo sostenible y de algunos modelos de A21L, a la vez que organizó, conjuntamente con los gobiernos locales, la primera conferencia en torno a la A21L en el país, donde se presentó la guía de A21L elaborada por el ICLEI (Gomila, 2001). Por ello, en el caso de Alemania, ante la ausencia de liderazgo por parte del Gobierno Federal, han sido los Estados Federales (Länder), quienes han asumido la difusión de la A21L (con la transferencia de información, la recopilación de buenas prácticas, la elaboración de documentación y manuales, etc.), llegando, en algunos casos, a financiar algunos proyectos piloto. Parece, que todo ello ha impulsado a que, en los últimos años, numerosas autoridades locales se vean inmersas en procesos de $\mathrm{A} 21 \mathrm{~L}$.

\subsubsection{Austria}

Austria, contaba con muchos de los factores que favorecian, a priori, la implantación de la A21L. Sin embargo, los procesos de A21L se desarrollaron con cierto retraso, que pudo ser debido, según Narodoslawsky (2001), a que en el discurso acerca de los contenidos del Desarrollo se dio prioridad al concepto de economía de 
mercado eco-social, frente al concepto de Desarrollo Sostenible. A pesar de ello, muchas comunidades austriacas se involucraron en ambiciosos programas que se basaron en los principios de Desarrollo Sostenible y que fueron presentados como Agendas 21 Locales, aunque no siempre tuviesen una conexión clara con ella.

En este marco, la actividad del gobierno austriaco en relación a la A21L fue muy limitada en los primeros años. Para atajar este retraso respecto a otros países europeos, se constituyó, en el año 2000, un Comité formado por los representantes de los nueve Länders, en temas de desarrollo sostenible coordinados por un órgano a nivel federal, que se encargará de evaluar las estrategias de Desarrollo Sostenible de los diferentes Länders. En este sentido, puede afirmarse que las Agendas 21 regionales han desempeñado un papel primordial en Austria, debido al gran porcentaje de municipios pequeños existente en Austria, que hace necesario que se dé un apoyo más firme por parte de los gobiernos regionales y por las características propias de una estrategia a nivel regional de este tipo, donde se aborda el desarrollo de una manera integral y a largo plazo, que le hace muy apropiada para acceder a la financiación de los Fondos Estructurales de la Unión Europea

En este contexto, el Gobierno Federal presentó, en abril de 2002, su Estrategia Nacional de Desarrollo Sostenible A Sustainable Future for Austria - The Austrian Strategy for Sustainable Development. Para coordinar la implementación de la Estrategia, se ha constituido el Comité para una Austria Sostenible (Committee for a Sustainable Austria, CSA), y se ha establecido un panel consultor de expertos (Forum for Sustainable Austria, FSA). Además, con el objetivo de fomentar el diálogo social e institucional, se han creado diversos foros como la Red Sostenible Austria o la mesa redonda Economía de Mercado Eco-Social. Por último, se aprobó en el verano de 2003 por el Consejo Regional de Ministros Ambientales un Programa de Acción Nacional en materia de Agenda 21.

\subsubsection{Francia}

Francia, no ha sido un país pionero en la implantación de la A21L, aunque ha desarrollado numerosas experiencias complejas y de gran riqueza. En 1993, con el fin de hacer realidad los compromisos adquiridos en la Cumbre de Río, se establecieron las primeras actuaciones materializadas en las llamadas Cartas de Ecología Urbana y Cartas del Medioambiente, que trataban de introducir el objetivo del Desarrollo Sostenible en la gestión local. En 1994, se creó el Comité 21, para ayudar a las corporaciones locales y a los ciudadanos a incorporar el Desarrollo Sostenible en sus actividades. Al año siguiente, en 1995, se creó la Comisión Francesa de Desarrollo Sostenible (CFDD), dependiente directamente del Primer Ministro, con la misión de definir las orientaciones de la Política Nacional de Desarrollo Sostenible. Sin embargo, esta Comisión no realizó actuaciones destacadas hasta que, en 1997, elaboró la 
Estrategia Francesa de Desarrollo Sostenible, que situó a la A21L entre sus prioridades. Un paso aún más definitivo, fue la creación, también en 1997, del Ministerio de Ordenación Territorial y Medioambiente, que permitió la integración de las Políticas ambientales con la Planificación territorial.

Por su parte, el Ministerio de Ecología y Desarrollo Sostenible (MEDD), es el encargado de financiar el desarrollo de la A21L, aunque las ayudas que reciben los Ayuntamientos sólo cubren una parte muy pequeña del presupuesto total de sus proyectos (Larrue et al., 2001). Asimismo, la Agencia de Medioambiente y Energía (L'ADEME), también concede cierta financiación a la que se pueden presentar los proyectos de A21L en temas más concretos como pueden ser la mejora de la eficiencia energética, la gestión de residuos, el transporte, la calidad ambiental, etc. Por último, es de esperar, que en los próximos años, gracias a la reciente incorporación de la A21L en la Política Nacional sobre Planificación Espacial, se produzca un incremento del número de iniciativas por parte de las autoridades locales (Larrue et al., 2001).

\subsubsection{Italia}

En 1993, el Ministerio de Medioambiente italiano elaboró el Plan Nacional de Desarrollo Sostenible y Agenda 21 Local (Piano nazionale per lo Sviluppo sostenibile in attuazione dell'Agenda 21). Sin embargo, a nivel local la respuesta fue más tardía, influyendo, probablemente, el gran número de municipios pequeños en los que se divide Italia. La Agenda 21, todavía no constituye un proceso plenamente desarrollado, aunque podemos encontrar ya a numerosas autoridades locales (municipios y provincias), que han comenzado a desarrollar sus Agendas 21 Locales (Khakee y Barbanente, 2003). Así, por ejemplo, en el año 2002, el centro de investigación FocusLab, en colaboración con la Asociación Italiana para la Agenda 21, realizó una encuesta que reflejó que más de 550 autoridades locales se encontraban comprometidas con la A21L.

Igualmente, ha sido muy notable la adhesión de las ciudades y pueblos italianos a la Carta de Aalborg, pasando de 50 ciudades en 1999, a más de 850 en el año 2003. En esta sorprendente evolución, han colaborado diversos organismos e instituciones, entre los que destaca la Red de Agendas 21 Locales de Italia y el Ministerio de Medio Ambiente, a través de la concesión de incentivos financieros a las autoridades locales que hayan firmado la Carta de Aalborg. Hay que destacar también el movimiento de A21L promovido por la Associazione Coordinamento Agenda 21 Locali Italiane, la experiencia a mayor escala de red nacional en Europa, que se constituyó con la Carta de Ferrara del año 2000. Otra institución relevante, la ANPA - Agenzia Nazionale per la Protezione dell'Ambiente, en la actualidad APAT (Agencia para la Protección del Medioambiente y para los Servicios Técnicos), realizó en colaboración con el Instituto 
Tabla 2. Estrategia y Financiación de la A21L.

\begin{tabular}{|c|c|c|}
\hline & Estrategia de Desarrollo Sostenible & Financiación \\
\hline Suecia & $\begin{array}{l}\text { Estrategia de Desarrollo Sastenible } \\
\text { (1995) }\end{array}$ & $\begin{array}{l}\text { Programa de Inversión Local (LIP) desde } \\
1998\end{array}$ \\
\hline Noruega & $\begin{array}{l}\text { National Strategy for Sustainable } \\
\text { Development (2002) }\end{array}$ & s.d. \\
\hline Dinamarca & $\begin{array}{l}\text { Shared Future Balanced } \\
\text { Development (2002) }\end{array}$ & $\begin{array}{l}\text { Fondos Verdes y Financiación del desa- } \\
\text { rrollo urbano }\end{array}$ \\
\hline Finlandia & $\begin{array}{l}\text { Elaboración de las opciones de } \\
\text { futuro de Finlandia: la implanta- } \\
\text { ción de la Agenda } 21 \text { en Finlandia }\end{array}$ & s.d. \\
\hline Reino Unido & $\begin{array}{l}\text { Estrategia Nacional de Desarrollo } \\
\text { Sostenible (1993) } \\
\text { Segunda Estrategia Nacional A } \\
\text { better quality of life (1999) }\end{array}$ & No \\
\hline Irlanda & $\begin{array}{l}\text { Sustainable Development: A } \\
\text { Strategy for Ireland (1997) }\end{array}$ & $\begin{array}{l}\text { Financiación para financiar la adopción de } \\
\text { sistemas de gestión ambiental y estableci- } \\
\text { miento de líneas de acción ambiental den- } \\
\text { tro de las A21L (desde 1997) }\end{array}$ \\
\hline Austria & $\begin{array}{l}\text { A Sustainable Future for Austria- } \\
\text { The Austrian Strategy for } \\
\text { Sustainable Development (2002) }\end{array}$ & s.d. \\
\hline Alemania & $\begin{array}{l}\text { Estrategia Nacional "Perspektiven } \\
\text { für Deutschland" (2002) }\end{array}$ & s.d. \\
\hline Holanda & Duurzame Daadkracht (2003) & $\begin{array}{l}\text { Programa para la Financiación de la Polí- } \\
\text { tica Ambiental Municipal (BUGM), el Pro- } \\
\text { grama para la Política Ambiental Nacional } \\
\text { (FUN) y Financiación para el Desarrollo } \\
\text { de la Política Ambiental Municipal (VOGM) }\end{array}$ \\
\hline Francia & $\begin{array}{l}\text { Estrategia Francesa de Desarrollo } \\
\text { Sostenible (1997) }\end{array}$ & $\begin{array}{l}\text { Outils de démarches menant à la réalisa } \\
\text { tion d'agendas } 21 \text { locaux (Financiación del } \\
\text { Ministerio de Ecología y Desarrollo Sos- } \\
\text { tenible, MEDD), Financiación de L'ADEME }\end{array}$ \\
\hline Italia & $\begin{array}{l}\text { Piano nazionale per lo Sviluppo } \\
\text { sostenible in attuazione } \\
\text { dell'Agenda } 21 \text { (1993) }\end{array}$ & $\begin{array}{l}\text { Financiación del Mnisterio de Medio } \\
\text { Ambiente y Premio a las ciudades } \\
\text { sostenibles italianas }\end{array}$ \\
\hline Portugal & $\begin{array}{l}\text { Estrategia Nacional de Desarrollo } \\
\text { Sostenible (2002) }\end{array}$ & No \\
\hline España & $\begin{array}{l}\text { Borrador de la Estrateia Nacional } \\
\text { de Desarrollo Sostenible (2002) }\end{array}$ & No \\
\hline
\end{tabular}

Fuente: Elaboración propia. 
de Investigación del Medio Ambiente de Italia una guía práctica para la A21L, Linee guida per le agende 21 locali (ANPA, 2000). La APAT, también ha elaborado una base de datos que recopila buenas prácticas europeas mediante el proyecto GELSO (GEstione Locale per la SOstenibilità). Por último, en lo que se refiere a la financiación, las ayudas destinadas desde el Ministerio de Medio Ambiente ascendieron a 12,9 millones de euros para 111 proyectos en el año 2000 y a 13,9 millones de euros para 60 proyectos, en el 2002. En suma, de acuerdo con todas estas actividades y programas realizados, se puede considerar que Italia, a pesar de que reaccionó tarde a la Cumbre de la Tierra, en los últimos años, se ha volcado con la Agenda 21 y el Desarrollo Sostenible, por lo menos a nivel de compromiso político.

\subsubsection{España}

En los últimos años, se han iniciado numerosos procesos de AL21 en el territorio español, siendo éste un fenómeno destacable porque se ha partido, la mayoría de las veces, de una total ausencia de políticas de sostenibilidad a nivel municipal. En esta dirección se lanzó, en el año 2002, desde el Gobierno Central, la Estrategia Española de Desarrollo Sostenible, abandonada, posteriormente, desde el año 2004.

En cualquier caso, los avances producidos en España en relación con la implantación de la A21L son significativos, más aún cuando no existía una cultura y una tradición medioambiental previa, en contraposición a otros países europeos, como Holanda o Dinamarca. Prueba de esta positiva evolución, es el incremento del número de firmantes españoles de la Carta de Aalborg, que en julio de 2005 alcanzaban casi los novecientos.

En este contexto, en los últimos años, se está empezando a apreciar un cambio significativo. Así, las Diputaciones y los Gobiernos Autónomos, han comenzado a desempeñar un papel primordial a la hora de promover su implantación, aunque las Agendas 21 Locales sean procesos de implementación local. De este modo, la mayoría de las Comunidades han emprendido la realización de un Diagnóstico Medioambiental y la oferta de líneas de financiación para los Ayuntamientos que quieran iniciar dichos procesos. Aunque hay otras regiones que han ido más allá y han elaborado sus propias Estrategias de Sostenibilidad como Navarra, País Vasco o Cataluña y están trabajando con grupos de Ayuntamientos, asesorándoles y dotándoles de instrumentos diversos tales como la formación de personal, la publicación de guías metodológicas o el diseño de indicadores, entre otros. Pero, también existen Comunidades, cuyos Gobiernos Autonómicos no están realizando apenas ninguna acción como es el caso de Extremadura, Comunidad Valenciana o Murcia (Echebarria y Aguado, 2003; Echebarria, et al., 2004; Galdós y Ruiz, 2005). 
Si analizamos los agentes participantes, en, prácticamente, casi todas las Comunidades Autónomas, los programas de A21L son competencia de los Departamentos de Medio Ambiente, a excepción de Cataluña, donde estas actividades se coordinan desde Presidencia, y Extremadura, donde se sitúan en la Consejería de Desarrollo Rural. Además, pueden existir otros Departamentos o Fundaciones, como es el caso de la Fundación Doñana 21 en Andalucía, o de DEYNA en Castilla y León, de FIDA en Madrid o de IHOBE en el País Vasco, que colaboren en tareas específicas.

Igualmente, las asociaciones o redes de municipios, tanto las creadas exclusivamente para el Desarrollo Sostenible (la Xarxa en Cataluña, la RECSA en Andalucía o Udalsarea en la CAPV, entre otras), como las Federaciones de Municipios y Provincias (Canarias, Castilla la Mancha o Murcia), también desempeñan un papel primordial, sobre todo, a la hora de poner en contacto a los diferentes Ayuntamientos y, favorecer, de este modo, la transferencia de información entre ellos. Otro elemento interesante es la creación de organismos que desempeñan funciones de asesoría o investigación como es el caso de los Consejos de Sostenibilidad en Andalucía, Baleares, Cantabria y Cataluña o la constitución de Foros de debate sobre Desarrollo Sostenible en Baleares y Canarias, que desempeñan similares funciones (Aguado y Echebarria, 2004; Brunet et al., 2005; Llamas et al., 2005).

\subsubsection{Portugal}

En Portugal, la respuesta a la A21L ha sido poco notable, pudiéndose achacar esta falta de iniciativas a la escasa tradición en la elaboración de políticas ambientales en el país. Y ello, a pesar de que Portugal asumió la Presidencia de la Unión Europea en la primera mitad de 1992, y como líder de la delegación de la Unión Europea en la Cumbre de Río trató de presentarse como un país con sólidas aspiraciones ambientales en su agenda política. Aún así, en una encuesta realizada en 1997, más de la mitad de los municipios portugueses no conocían la Agenda 21 (Carter et al., 2000).

Dentro del Gobierno de la República, ha sido el Instituto Portugués de Ecología (INPECO), el que ha asumido las competencias en la promoción de la A21L, aunque no ha establecido fondos específicos para su implantación. En cualquier caso, en el año 2002, se publicó la Estrategia Nacional de Desarrollo Sostenible y fue sometida a consulta pública. Pese a ello, la impresión general que se obtiene al analizar el caso portugués es que no existe un apoyo fuerte por parte del Gobierno de la República, que los municipios, en su mayoría, no están desarrollando la A21L, que las ONGs están ejerciendo un papel muy pasivo y que la ciudadanía, prácticamente, no conoce este programa ni sus repercusiones, (Carter et al., 2000). 
Tabla 3. Agentes Institucionales en Europa.

\begin{tabular}{|c|c|c|}
\hline & $\begin{array}{l}\text { Comité Nacional de } \\
\text { A21/Örgano Coordinador }\end{array}$ & Principales Agentes a nivel nacional \\
\hline Suecia & $\begin{array}{l}\text { National Committee for } \\
\text { Agenda } 21 \text { (1995) } \\
\text { Swecol, Centro Sueco para la } \\
\text { Sostenibilidad Ecológica (1999) }\end{array}$ & $\begin{array}{l}\text { Ministro de Medioambiente, Asociación de } \\
\text { Autoridades Locales, Asociación para la } \\
\text { Agenda } 21 \text { y el Desarrollo Sostenible }\end{array}$ \\
\hline$\overline{\text { Noruega }}$ & $\begin{array}{l}\text { Órgano de coordinación de la } \\
\text { A21L (1997) } \\
\text { Comité Verde (2003) }\end{array}$ & $\begin{array}{l}\text { The Royal Norwegian Ministry of Foreign } \\
\text { Affairs, Ministerio de Mecioambiente y Energía, } \\
\text { Ministerio de Finanzas, Oficina del Primer } \\
\text { Ministro }\end{array}$ \\
\hline Dinamarca & $\begin{array}{l}\text { Minsterio de Medioambiente } \\
\text { y Energía }\end{array}$ & $\begin{array}{l}\text { Ministerio de Asuntos Urbanos y Vivienda, } \\
\text { Ministerio de Medio Ambiente y Planificación } \\
\text { Territorial, la Asociación Nacional de Autorida- } \\
\text { des Locales y la Asociación Danesa de Conda- } \\
\text { dos y Concejos, Ministerio de Asuntos Urbanos } \\
\text { y Vivienda }\end{array}$ \\
\hline Finlandia & $\begin{array}{l}\text { Comisión Nacional de Agenda } 21 \\
\text { dirigida por el Primer Ministro }\end{array}$ & $\begin{array}{l}\text { Comisión Nacional de Desarrollo Sostenible } \\
\text { (1993), Asociación de Regiones y Autoridades } \\
\text { Locales Finlandesas (AFLRA) }\end{array}$ \\
\hline Austria & $\begin{array}{l}\text { Committee for a Sustainable } \\
\text { Austria (CSA) }\end{array}$ & $\begin{array}{l}\text { Forum for Sustainable Austria (FSA), } \\
\text { Consejo Regional de Ministros Ambientales }\end{array}$ \\
\hline Reino Unido & $\begin{array}{l}\text { Federación de Gestión del Gobierno } \\
\text { Local (LGMB) que ha derivado en la } \\
\text { Agencia de Mejora y Desarrollo } \\
\text { (I\&DeA) }\end{array}$ & $\begin{array}{l}\text { Primer Ministro, Asociación de Autoridades } \\
\text { Locales (LGA) y Department of Environment, } \\
\text { Transport and the Regions (DETR) }\end{array}$ \\
\hline Irlanda & Department of the Environment & $\begin{array}{l}\text { Department of the Environment and Local } \\
\text { Government, Comités de Política Estratégica } \\
\text { (SPCs), Equipos de Desarrollo Comarcal y } \\
\text { Municipal (CDBS), Instituto de Administración } \\
\text { Pública (IPA) }\end{array}$ \\
\hline Alemania & $\begin{array}{l}\text { Agencia Federal de Medioambiente } \\
\text { (Umweltbundesamt, UBA) }\end{array}$ & $\begin{array}{l}\text { Ministerio Federal del Medio Ambiente, Minis- } \\
\text { terio Federal de Investigación, Oficina de Can- } \\
\text { ciller Federal (Bundeskanzleramt), Consejo } \\
\text { Científico del Gobierno Federal sobre Cambios } \\
\text { Medioambientales Globales (Wissenschaftlicher } \\
\text { Beirat der Bundesregierung Globale } \\
\text { Umweltverändertingen, WBGU) }\end{array}$ \\
\hline Holanda & Comisión Nacional de A21L (1994) & $\begin{array}{l}\text { Ministerio de Medioambiente (VROM), la Aso- } \\
\text { ciación Holandesa de Municipios (VNG) y el } \\
\text { Comité Nacional de Cooperación Internacional } \\
\text { y Desarrollo Sostenible (NCDO) }\end{array}$ \\
\hline Francia & Comité 21 (1994) & $\begin{array}{l}\text { Comisión Francesa de Desarrollo Sostenible } \\
\text { (Commission Française du Développement } \\
\text { Durable, CFDD), Ministerio de Ecologia y } \\
\text { Desarrollo Sostenible (MEDD), Agencia de } \\
\text { Medioambiente y Energía (L'ADEME) }\end{array}$ \\
\hline
\end{tabular}




\begin{tabular}{lll}
\hline & & \\
& Comité Nacional de & Principales Agentes a nivel nacional \\
A21/Örgano Coordinador & \\
\hline Italia & Associazione Coordinamento & FocusLab, Asociación Italiana para la Agenda \\
& Agenda 21 Local Italiane & 21, Red de las Agendas 21 Locales de Italia, \\
& & Ministerio de Medio Ambiente, ANPA-Agenzia \\
& Nazionale per la Protezione dell'Ambiente que & ha derivado en la APAT \\
\hline Portugal & Instituto Portugués de & Ministerio de Asuntos Urbanos, Planificación \\
& Ecología (INPECO) & Territorial y Medio Ambiente \\
\hline España & No & Ministerio de Medio Ambiente, Federación \\
& & Espanola de Provincias y Municipios (FEMP) \\
\hline
\end{tabular}

Fuente: Elaboración propia.

\subsubsection{Irlanda}

El gobierno irlandés comenzó a incluir el Desarrollo sostenible entre sus objetivos en 1994, con el Plan de Desarrollo Nacional 1994-1999. Aunque no será hasta el año 1997, cuando apruebe su Estrategia específica para la consecución de un Desarrollo Sostenible, mediante la elaboración del documento titulado Sustainable Development: A Strategy for Ireland.

Simultáneamente a este incremento de la concienciación política respecto al Desarrollo Sostenible, la A21L comenzó a ser percibida como un instrumento útil para la consecución de dicho objetivo. De ahí, que en 1995, el Departamento de Medioambiente publicase una guía sobre la A21L dirigida a las autoridades locales, aunque a partir de 1997, con motivo de la revisión de la Cumbre de Río, tras la Conferencia de "Río + 5", el gobierno solicitará oficialmente a las autoridades locales que completen sus Agendas 21 Locales, destacando la necesidad de nombrar técnicos de A21L. Por ello, cada autoridad local en Irlanda tiene designado un Técnico en A21L. Sin embargo, estos nombramientos no han dado todavía sus frutos, ya que los avances conseguidos se limitan, por ahora, a la introducción de la variable ambiental en el conjunto de las actividades que realizan las instituciones (Kelly et al., 2004).

En todo caso, el hecho más relevante a destacar en el ámbito irlandés ha sido la creación de Comhar, the National Sustainable Development Partnership, en Febrero de 1999, establecido como un foro nacional multisectorial en torno a los aspectos clave del Desarrollo Sostenible (Mullally, 2001). Por último, hay que destacar la financiación que se ha venido concediendo, desde 1997, para el desarrollo de diversos proyectos, por parte de los municipios y las ONGs, relacionados con la adopción de sistemas de gestión ambiental y el establecimiento de lineas de acción ambiental en el marco de la A21L. 


\subsection{Sintesis Comparativa de la Situación de la Agenda 21 Local en Europa}

En la Tabla 2, se muestra la relación de países que han desarrollado Estrategias de Desarrollo Sostenible. Se incluye también a Holanda y Dinamarca, que aunque en la fecha de elaboración de la tabla no habían presentado todavía sus estrategias de Desarrollo Sostenible, han acometido otro tipo de estrategias de política ambiental, bastante similares a las del resto de los países. La articulación de financiación por parte de los gobiernos nacionales es uno de los factores que más influye en la difusión de la A21L. En efecto, se puede achacar el despegue de la A21L en Francia e Italia a la financiación concedida por los gobiernos nacionales, a pesar de que estas ayudas sean pequeñas, e, incluso, podríamos decir que casi testimoniales.

Otro aspecto a destacar, es el número y naturaleza de los agentes comprometidos con la A21L. Por un lado, es primordial, como se muestra en la Tabla 3, la existencia de algún órgano coordinador, que asuma las competencias de organizar y dirigir las acciones de promoción de la A21L a nivel nacional. Por otro lado, también resulta substancial la participación del mayor número posible de organizaciones e instituciones. En todo caso, parece, especialmente interesante el hecho de que estas instituciones no se reduzcan a las meramente ambientales, ya que ello puede servir para dar una mayor transversalidad a la acción de la A21L. Sin embargo, resulta evidente el predominio de las entidades de carácter medioambiental o ecológico y, de hecho, el Ministerio de Medio Ambiente suele asumir el papel de promotor en la mayoría de los paises (Irlanda, Portugal, España, Suecia, etc.). Otro indicador que se refleja también en la Tabla 3 es la presencia o participación de las Asociaciones de Municipios (Suecia, Dinamarca, Reino Unido, etc.), que pueden cumplir el papel de intermediarios entre los gobiernos nacionales y locales.

Por último, dentro del marco europeo, se puede concluir la existencia de tres grupos de países (Véase Tabla 4):

- El primer grupo, está formado por aquellos países que hemos denominado pioneros (Suecia, Reino Unido y Holanda), caracterizados por su rápida respuesta a la llamada de la Cumbre de Río de 1992, y, por el resto de los países escandinavos (Dinamarca, Noruega y Finlandia), que aunque empezaron más tarde que los anteriores, han manifestado un notable compromiso con los postulados del Desarrollo Sostenible.

- El segundo grupo, lo componen Alemania y Austria, con una respuesta más tardía que los anteriores y donde el seguimiento de la A21L, ha sido bastante escaso.

- El tercer grupo, lo conforman, fundamentalmente, los países mediterráneos (Italia, Portugal, España y Francia) junto con Irlanda, donde el interés por la A21L data de finales de los años noventa. 
Tabla 4. Impulso otorgado a la Agenda 21 Local en Europa.

\begin{tabular}{|c|c|c|c|}
\hline & $\begin{array}{l}\text { Respuesta Temprana } \\
\text { (antes del año 1994) }\end{array}$ & $\begin{array}{l}\text { Repuesta Media } \\
\text { (1994-1998) }\end{array}$ & $\begin{array}{l}\text { Respuesta Tardia } \\
\text { (después del año 1998) }\end{array}$ \\
\hline Impulso Alto & $\begin{array}{l}\text { Suecia } \uparrow \\
\text { Holanda } \pi\end{array}$ & $\begin{array}{l}\text { Noruega } \pi \\
\text { Dinamarca } \uparrow\end{array}$ & Irlanda $\nearrow$ \\
\hline $\begin{array}{l}\text { Impulso } \\
\text { Medio }\end{array}$ & لע Reino Unido & Finlandia $\rightarrow$ & $\begin{array}{l}\text { España } \uparrow \\
\text { Italia } \uparrow \\
\text { Francia } \uparrow\end{array}$ \\
\hline Impulso Bajo & & $\begin{array}{l}\text { Austria } \rightarrow \\
\text { Alemania } \rightarrow\end{array}$ & Portugal $\rightarrow$ \\
\hline \multicolumn{4}{|c|}{$\begin{array}{l}\text { TTrayectoria muy positiva } \\
\lambda_{\text {Trayectoria positiva }} \\
\vec{\forall}_{\text {No hay avances significativos }} \\
\text { Trayectoria negativa }\end{array}$} \\
\hline
\end{tabular}

Fuente: Elaboración propia.

\section{Conclusiones}

El artículo tiene por objeto presentar un análisis de la implantación de la A21L en Europa. Constatamos, el gran auge que se estáa dando a la difusión de los procesos de A21L a través del planeta. No obstante, se confirman también las grandes diferencias regionales en el ritmo de desarrollo y enfoque de los programas de A21L.

Así, el mayor esfuerzo, que corresponde a aquellos países con una larga tradición en política ambiental y con mayor autonomía de gobierno local, se localizan en el grupo de cabeza (Suecia, Holanda, Dinamarca, Noruega y Finlandia), a excepción de Alemania. Resulta curioso que en Alemania, a pesar de que se dan estas dos condiciones, no se hayan promovido acciones relativas a la A21L por parte del Gobierno Central hasta el año 1998. Por el contrario, sorprende el pronto compromiso del Reino Unido, país con escasa tradición en políticas medioambientales y con limitada autonomía por partes de las autoridades locales. En el caso de los países del arco mediterráneo, su gran extensión, el elevado número de municipios de pequeña dimensión, su escasa tradición ambientalista y la prioridad otorgada a las variables económicas, impuesta por el Tratado de Maastricht, nos ayudan a comprender la ralentización de su compromiso con la Agenda 21 Local. No obstante, hemos de señalar que las grandes diferencias iniciales en cuanto al grado de desarrollo de las Agendas 21, se han ido suavizando en los últimos años y, de hecho, pensamos que se está produciendo un cierto cansancio entre los países pioneros, que están tendiendo a relegar estos procesos a un segundo plano, mientras que en los países medi- 
terráneos, se está dando el efecto contrario, pudiéndose apreciar un fuerte impulso respecto a la Agenda 21 Local.

Finalmente, en términos generales, es destacable la creciente concienciación mundial respecto a que los objetivos de las estrategias de desarrollo sostenible sólo podrán alcanzarse con la puesta en marcha de procesos participativos de debate, planificación y acción como la Agenda 21. La articulación de financiación por parte de los gobiernos nacionales (o regionales en países más descentralizados) y la existencia de algún órgano coordinador, que asuma las competencias de organizar y dirigir las acciones de promoción de la A21L a nivel nacional, son dos de los factores que más influyen en la difusión de la A21L. Sobre estas bases, la puesta en marcha de densas redes de colaboración público-privada, con la participación del mayor número posible de organizaciones e instituciones, suele resultar un impulsor de primer orden.

\section{Bibliografía}

Aall, C. (2001): Norway Local Agenda 21 as a means of interpreting and achieving Sustainable Production and Consumption. En Lafferty, W.M. (ed) Sustainable Communities in Europe, London, Earthscan, p. 266-304.

Adolfsson, S. (2002): Local Agenda 21 in Four Swedish Municipalities: A Tool towards Sustainability? Journal of Environmental Planning and Management, 45 (2), 219244.

Aguado, I. y Echebarria, C. (2004): El gasto medioambiental en las Comunidades Autónomas y su relación con la Agenda Local 21: Estudio mediante el empleo del análisis de correspondencias. Estudios Geográficos, 65 (255), 195-228.

Bond, A.J., Mortimer, K.J. y Cherry, J. (1998): The Focus of Local Agenda 21 in the United Kingdom. Journal of Environmental Planning and Management, 41 (6), 767-776.

Brunet Estarellas, P.J. Almeida García, F. y Coll López, M. (2005): Agenda 21: Subsidiariedad y cooperación a favor del desarrollo territorial sostenible. Boletin de la Asociación de Geógrafos Españoles, 39, 423-446.

Carter, N., Nunes Da Silva, F. y Magalhaes, F. (2000): Local Agenda 21: Progress in Portugal. European Urban and Regional Studies, 7 (2), 181-186.

Church, C. y Young, S. (2001): The United Kingdom. Mainstreaming, mutating or expiring. En Lafferty, W.M. (ed) Sustainable Communities in Europe, London, Earthscan, p. 107-129.

CNUMAD (1992): La Agenda Local 21 (en línea). Nueva York: Naciones Unidas. <http://www.un.org/esa/sustdev/agenda2 1sp/index.htm> (Consulta: 09.05.2004)

Coenen, F. (2001): The Netherlands: Probing the essence of LA21 as a value-added approach to sustainable development and local democracy. En Lafferty, W.M. (ed) Sustainable Communities in Europe, London, Earthscan, p. 153-179.

Comité 21. (2003): Territoires et Développement durable. Tomo. II (en 
línea). París, Comité français pour l'environnement et le développement durable. $<$ http://www.comite21.org/ guides /guide_territoire_dev_durable2.pdf> (Consulta: 11.07.2006)

Echebarria, C. y Aguado, I. (2003): La Agenda Local 21 como instrumento de sostenibilidad: la experiencia española. Revista Española de Estudios Agrosociales $y$ Pesqueros, 199, 61-91.

Echebarria, C., Barrutia, J.M. y Aguado, I. (2004): Local Agenda 21: Progress in Spain. European Urban and Regional Studies, 11 (3), 273-281.

Eckerberg, K, Edström, C. y Storm, L. (2003): Lokal Agenda 21 i ett politikerperspektiv (documento de trabajo no publicado). Statsvetenskapliga institutionen, Uneå universitet.

Font, N. y Subirats, J. (2000): El reto de la sostenibilidad local en España. En Font, N. y Subirats, J. (eds) Local y sostenible. La Agenda 21 Local en España. Barcelona, Icaria, p. 93-110.

Frank, P., Møller, D.R. y Jørgensen, V. (eds) (2000): Local Agenda 21 in Denmark State of implementation in late 1998 (en línea). Copenhagen: Ministry of Environment and Energy. $<\mathrm{http}: / /$ pa.dk/Topmenuen/Publikationer $/ \mathrm{Andre}-\mathrm{sprog} / 2000 /$ agenda21_status_rapport_98_99_uk.pdf> (Consulta: 29.03.2005)

Galdós Urrutia, R. y Ruiz Urrestarazu, E. (2005): El camino hacia la sostenibilidad en el País Vasco. La Agenda 21 en VitoriaGasteiz. Geographicalia, 48, 77-99.

Gomila, M.F. (2000): La Agenda 21 Local en Europa: un análisis comparado. , N. y Subirats, J. (eds) Local y sostenible. La Agenda 21 Local en España, Barcelona, Icaria, p. 29-64.

Gram-Manssen, K. (2000): Local Agenda 21: Traditional Gemeinschaft or Late-Modern
Subpolitics?. Journal of Environmental Policy E Planning, 2 (3), 225-235.

Heidbrink, K. y Paulus, S. (2000): Strategies for sustainable development in the thicket of national planning processes. From convergent concepts to coherent actions in development cooperation. Eschborn (Germany), Deutsche Gesellschaft für Technische Zusammenarbeit (GTZ) GmbH.

Hewitt, N. (1998): Guia Europea para la planificación de las Agendas 21 Locales. Cómo implicarse en un plan de acción ambiental a largo plazo bacia la sostenibilidad. Bilbao, Bakeaz.

Holm, J. y Wambui Kamara, M. (2001): Denmark The participatory and consensus-seeking approach of the Danish LA21. En Lafferty, W.M. (ed) Sustainable Communities in Europe, London, Earthscan, p. 58-82.

ICLEI (1997): Local Agenda 21 Survey. A Study of Responses by Local Autborities and their National and International Association to Agenda 21 (en línea). Nueva York: UNDESA. < h t p : / / w w w i cle i.org/ LA21/LA21rep.htm> (Consulta: 29.06.2004)

ICLEI (2002): Local Agenda 21 Survey: $A$ Study of Responses by Local Authorities and their National and International Associations for Agenda 21. Canada, ICLEI.

Jonas, A.E.G., While, A. y Gibbs, D.C. (2004): State modernisation and local strategic selectivity after Local Agenda 21: evidence from three northern English localities. Policy \& Politics, 32 (2), 151-168.

Kelly, R., Sir, L. y Ratcliffe, J (2004): Futures thinking to achieve sustainable development at local level in Ireland. Foresight, 6 (2), 80-90. 
Khakee, A. (2002): Assessing institutional capital building in local agenda 21 process in Göteborg. SAGE Public Administration Abstracts, 29 (3), 309-456.

Khakee, A. y Barbanente, A. (2003): Negotiative land-use and deliberative environmental planning in Italy and Sweden. International Planning Studies, 8 (3), 181-200.

Lafferty, W.M. (2001): Introduction. En Lafferty, W.M. (ed) Sustainable Communities in Europe, London, Earthscan, p. 1-14.

Lafferty, W.M. y Eckerberg, K. (eds) (1998): From the Earth Summit to Local Agenda 21: Working Towards Sustainable Development. London, Earthscan.

Larrue, C., Emelianoff, C., Di Pietro, F. y Héland, L. (2001): France. LA21: A new tool for sustainable policies? En Lafferty, W.M. (ed) Sustainable Communities in Europe, London, Earthscan, p. 180-205.

Llamas, R., García, V.J. y López, F.J. (2005): Un análisis institucional de la implantación de la Agenda Local 21 por los ayuntamientos españoles. Revista Europea de
Dirección y Economia de la Empresa, Vol. 14 (3), 9-40.

Löffler, P. y Payne, A. (eds) (1999): An initial evaluation of the European Sustainable Cities \& Towns Campaign (en línea). Bruselas: European Sustainable Cities \& Towns Campaign. <http://www.sustainable-cities.org $>$ (Consulta: 14.03.2005)

Mullally, G. (2001): Ireland. Starting late: Building institutional capacity on the reform of sub-national governance?. En Lafferty, W.M. (ed) Sustainable Communities in Europe, London, Earthscan, p. 130-152.

Narodoslawsky, M. (2001): A regional approach to sustainability in Austria. International Journal of Sustainability in Higber Education, 2 (3), 226-237.

Niemi-Iilahti, A. (2001): Finland. In search of new implementation pattern. En Lafferty, W.M. (ed) Sustainable Communities in Europe, London, Earthscan, p. 40-57.

Wild, A. y Marshall, R. (1999): Participatory practice in the context of local agenda 21 : A case study evaluation of experience in three English local authorities. Sustainable Development, 7 (3), 151-162. 